\title{
Cytokines of the IL-6 family in the modulation of carbohydrate and lipid metabolism in patients with metabolic syndrome
}

\author{
Daria Skuratovskaia \\ IKBFU, Kaliningrad, Russia \\ DSkuratovskaya@kantiana.ru \\ Elena Kirienkova \\ IKBFU, Kaliningrad, Russia \\ elenamed@list.ru
}

\author{
Alexandra Komar \\ IKBFU, Kaliningrad, Russia \\ alexandkomar@gmail.com \\ Egor Shunkin \\ IKBFU, Kaliningrad, Russia \\ egor.shunkin@gmail.com
}

\author{
Maria Vulf \\ IKBFU, Kaliningrad, Russia \\ mary-jean@yandex.ru \\ Larisa Litvinova \\ IKBFU, Kaliningrad, Russia \\ larisalitvinova@yandex.ru
}

\begin{abstract}
According to the world community, metabolic complications are associated with inflammation in obesity. The cytokines of the interleukins (IL) - 6 family consist of IL-6, IL11, IL-27 and have similar, but individual effects, such as the regulation of liver damage, the balance between regulatory and effector $T$ cells and metabolic regulation. The antiinflammatory and regenerative activities of IL-6 are usually mediated by the classical signaling regimen, while the proinflammatory responses in many pathological conditions include trans-signaling. The aim of the study was to assess the role of the cytokines of the IL-6 family: IL-6, sIL-6Ra, gp130 / sIL-6Rb, IL-11 in the regulation of carbohydrate (glucose level) and lipid (cholesterol (Hol), high-density lipoprotein (HDL), low-density lipoprotein (LDL), triglycerides (TR)) exchanges in serum / plasma in patients with metabolic syndrome (MetS). This fact may indicate the protective role of sIL-6Ra transsignaling and protective role of IL-6 signal inhibition due to high levels of sgp130 / sIL-6Rb in relation to metabolic disorders in obesity patients.
\end{abstract}

Keywords - IL-6, sIL-6Ra, gp130 / sIL-6Rb, IL-11, metabolic syndrome, cholesterol, glucose

\section{Introduction}

Obesity and accompanying complications (type 2 diabetes mellitus (type 2 diabetes), diabetic foot, atherosclerosis, nonalcoholic fatty liver disease (NAFLD), steatohepatitis, etc.) occupy a leading position among the causes of mortality [1]. The main signs of metabolic syndrome (MetS) are arterial hypertension, central obesity, hyperglycemia and atherogenic dyslipidemia, and concomitant signs are liver steatosis, polycystic ovary syndrome and hyperuricemia [2]. The main long-term complications of MetS are type 2 diabetes, atherogenesis, and cognitive impairment [2]. According to the world community, metabolic complications are associated with inflammation in obesity. Chronic subclinical inflammation contributes to the lipid and carbohydrate metabolism disorders, and its local foci lead to dysfunction of different tissues (adipose tissue, hepatic, vascular endothelium, etc.).

Initially, it was believed that IL- 6 is assigned to proinflammatory cytokines and is a predictor of type 2 diabetes, secreted by TNF-a-mediated manner [3; 4]. IL-6 is known to mediates adverse metabolic effects, promotes insulin resistance and worsens glucose homeostasis [4; 5]. However, the role of IL- 6 in potentiation of metabolic disorders is still controversial. Different types of cells involved in the regulation of glucose homeostasis respond differently to IL-6. Many experiments have shown that IL- 6 can potentiate insulin resistance in hepatocytes, but the results on adipocytes and skeletal muscles are contradictory/inconsistent $[3 ; 6]$.

The interleukin (IL) - 6 family cytokines is a group of cytokine consisting of IL-6, IL-11, IL-27 and belong to the same family, because the receptor complex of each cytokine contains two (for IL-6 and IL-11) or one molecule (for all other cytokines) of the gp130 signaling receptor subunit [6]. Cytokines of the IL-6 family have similar, but individual effects, such as the regulation of liver damage, the balance between regulatory and effector $\mathrm{T}$ cells, and metabolic regulation [6].

IL-6 realizes its effects through the membrane-bound receptor of IL-6R, which can exist in a soluble form (sIL-6R), the latter lacks the transmembrane and cytoplasmic domains. The main sources of sIL-6R are hepatocytes and immune cells. IL-6 binds to IL-6R or sIL-6R and initiates subsequent signaling through interaction with gp130 [3; 6]. The IL-6 / sIL-6R complex acts as an agonist of gp130-mediated IL-6 signaling, which is called trans-signaling. This type of signal transmission expands the spectrum of potential IL-6 targets due to the ubiquitous expression of gp130. However, gp130 can also be in soluble form and inhibit IL-6 trans-signaling without affecting the classical type of signal transmission [6]. Soluble gp130 (sgp130) is found in the bloodstream of healthy people by buffering the systemic response to circulating IL-6 $[6 ; 7]$.

The anti-inflammatory and regenerative activities of IL-6 are usually mediated by the classical signaling regimen, while the pro-inflammatory responses in many pathological conditions include trans-signaling.

In this regard, the purpose of the study was to assess the role of the IL-6 family cytokines: IL-6, sIL-6Ra, gp130 / sIL$6 \mathrm{Rb}, \mathrm{IL}-11$ in the regulation of components carbohydrate and lipid metabolism (glucose level, cholesterol (Hol), highdensity lipoprotein (HDL), low-density lipoprotein (LDL), triglycerides (TR)) in serum / plasma in patients with metabolic syndrome (MetS).

\section{Methods and Materials}

This study included 118 patients with MetS (53 men and 65 women, $48(41-53)$ years, BMI $=46.25(41.48-54.68)$ $\mathrm{kg} / \mathrm{m}^{2}$ ) who were admitted to the Regional Clinical Hospital. 
All patients were diagnosed with abdominal obesity. The presence of MetS was confirmed based on a detailed clinical and instrumental examination using the World Health Organization diagnostic criteria (Swarup S, Zeltser R, 2020). The control group consisted of healthy donors with normal anthropometric and biochemical parameters (23 men and 17 women, $35(30.5-49.5)$ years, BMI $=23.4(20.9-24.9)$ $\mathrm{kg} / \mathrm{m}^{2}, \mathrm{n}=40$ ).

All study participants provided informed consent to participate in a research study. The study was carried out in accordance with the World Medical Association (WMA) Declaration of Helsinki (2000) and the Protocol to the Convention on Human Rights and Biomedicine (1999). The study protocol was approved by the Local Ethical Committee of the Innovation Park of the Immanuel Kant Baltic Federal University (Protocol No. 4 from October 23, 2013).

Mediators of carbohydrate (glucose (GL)), and lipid metabolism (cholesterol (Hol), high-density lipoprotein (HDL), low-density lipoprotein (LDL), triglycerides (TR), Creactive protein (CRP)) were measured on a CA-180 automatic biochemical analyzer (Furuno Electric Co., Ltd, Japan).

Quantitative determination of IL-6, sIL-6Ra, gp130/sIL$6 \mathrm{Rb}$, IL-11 was evaluated by flow fluorimetry on an analyzer «Bio-Plex Protein Assay System» (Bio-Rad, USA) using BioPlex Pro ${ }^{\text {TM }}$ Human Inflammation Panel 1, 37-Plex (Bio-Rad, USA). Verification of quantitative indicator distribution normality was carried out using the Shapiro-Wilk test. For detecting statistically significant differences between groups, a pairwise analysis was performed using the nonparametric Mann-Whitney criterion for independent groups was used. Correlations between the studied indices were determined using Spearman correlation analysis. Differences were considered significant at the level of $\mathrm{p}<0.05$. All statistical analyses have been performed in GraphPad Prism 8 (USA).

\section{Result and Discussion}

\section{Biochemical parameters of carbohydrate and lipid metabolism}

Ectopic lipid deposition, chronic inflammation, disorder carbohydrate and lipid metabolism are characteristics of the metabolic syndrome (MetS) $[1 ; 2]$. The concept of metabolic syndrome combines the presence of obesity and at least two concomitant signs of metabolic disorders, according to the criteria for determining MetS of the World Health Organization [1]. The study revealed the disorder of carbohydrate and lipid metabolism in patients with MetS. glucose, cholesterol, LDL, triglycerides serum levels in patients with MetS were higher compared to controls ( $p$ $<0.05$ ), and the HDL levels were lower than control values ( $p$ $<0.05$ ) (Table 1).

TABLE 1. BIOCHEMICAL PARAMETERS OF CARBOHYDRATE AND LIPID METABOLISM IN BLOOD SERUM IN THE PATIENT GROUPS

\begin{tabular}{|c|c|c|c|}
\hline $\begin{array}{c}\text { Parameters } \\
(\mathrm{mmol} / \mathrm{l})\end{array}$ & $\begin{array}{c}\text { Control, } \\
\mathbf{n}=\mathbf{4 0}\end{array}$ & $\begin{array}{c}\text { Patients with MetS, } \\
\mathbf{n}=\mathbf{1 1 8}\end{array}$ & p-value \\
\hline Glucose & $5.09(4.66-5.36)$ & $6.50(5.40-9.33)$ & $\mathrm{p}=0.0011^{*}$ \\
\hline HOL & $4.42(3.80-5.17)$ & $5.29(4.34-5.96)$ & $\mathrm{p}<0.0001^{*}$ \\
\hline HDL & $1.46(1.05-1.71)$ & $1.16(0.96-1.34)$ & $\mathrm{p}=0.0140^{*}$ \\
\hline LDL & $2.19(1.65-2.47)$ & $2.79(2.25-3.38)$ & $\mathrm{p}=0.0002^{*}$ \\
\hline
\end{tabular}

\begin{tabular}{|c|c|c|c|}
\hline TR & $0.86(0.65-1.37)$ & $1.85(1.27-2.44)$ & $\mathrm{p}<0.0001^{*}$ \\
\hline CRP & $3.96(1.56-8.83)$ & $9.31(7.37-14.15$ & $\mathrm{p}=0.0003^{*}$ \\
\hline
\end{tabular}

Note: the significance is determined using the Mann-Whitney criterion for two independent samples $(*-\mathrm{p}<0.05)$.

\section{Pro-inflammatory mediators}

Obesity inflammation plays an important role in the development of MetS and other complications CRP is an inflammatory biomarker and refers to acute phase proteins. Elevated CRP levels are a companion to metabolic disorders, in particular MetS [8; 9]. In our study, the CRP level in patients with MetS (9.31 (7.37 - 14.15) pg / ml) was higher than the control $(3.96(1.56-8.83) \mathrm{pg} / \mathrm{ml})(\mathrm{p}<0.05)$, which indicates the presence of systemic inflammation in this category of patients (Table 1).

CRP is formed and released from the liver by the action of the cytokines IL-1 and IL-6 [9]. In our IL-6 study, plasma levels in patients with MetS (4.59 (3.99 - 6.59) pg / ml) exceeded the control of $1.45(0.55-6.59) \mathrm{pg} / \mathrm{ml}(\mathrm{p}<0.01)$ (Figure 1). However, IL-6 levels were negatively correlated with plasma CRP levels in patients with MetS $(\mathrm{r}=-0.522, \mathrm{p}$ $<0.05)$.
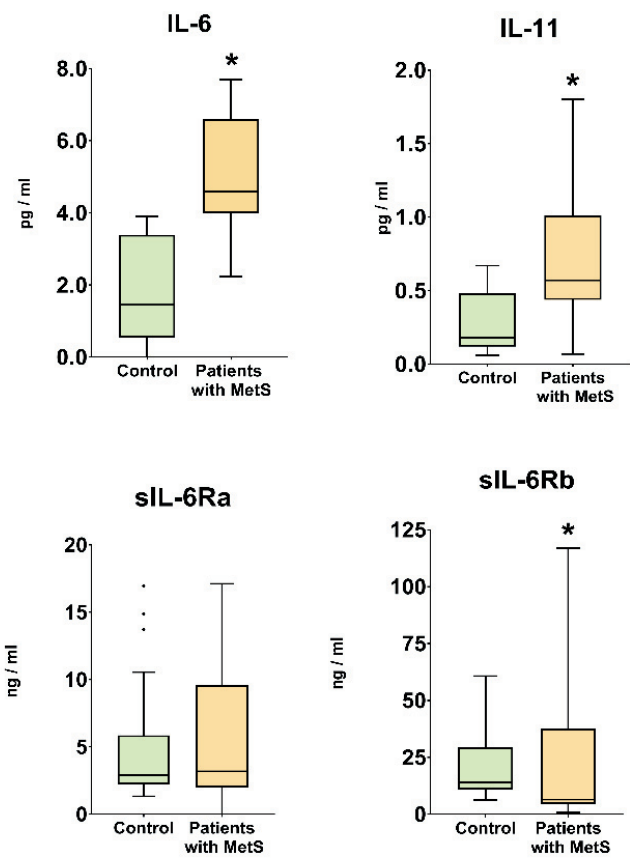

Fig. 1. The content of pro-inflammatory mediators in the blood plasma of patients with metabolic syndrome

IL-6 is usually not expressed constitutively, but its expression is intensively induced by a spectrum of stimuli, such as viral and bacterial infections, pro-inflammatory cytokines, oxidative stress, etc. [10]. IL-6 was initially considered a pro-inflammatory cytokine. There is an opinion that IL-6 and CRP belong to markers of type 2 diabetes [9; 10]. In our study, an increase in IL-6 level was positively correlated with an increase in BMI $(r=0.56, p<0.05)$ and an increase in glucose concentration $(r=0.50, p<0.05)$ in the blood serum of patients with MetS (Fig. 2). The observation that serum IL-6 levels correlate with obesity, impaired carbohydrate and lipid metabolism suggests that IL-6 is associated with the development of metabolic diseases. 
However, there is increasing evidence of antiinflammatory activity of IL-6. Mice with IL-6 deficiency develop obesity in adulthood, liver inflammation, and systemic insulin resistance [3]. In accordance with these observations, elevated cholesterol and triglyceride levels, accompanied by weight gain, were observed in patients who were treated with IL-6 inhibitors [3; 10]. Thus, the association between IL-6 and the progression of metabolic disorders in obesity may simply reflect an attempt to counteract the chronic inflammation caused by other inflammatory mediators.

The explanation may lie in a change in the signaling of IL6 in conditions of chronic inflammation in obesity. The scientific periodicals describe the properties of IL-6 dependent signaling in the regulation of metabolic parameters in obesity. sIL-6R binds IL-6 and prolongs the half-decay of IL-6 in animal models [11], enhancing regenerative processes in the liver. In addition, it was shown that the complex of IL- 6 and sIL-6R internalizes much less efficiently than IL-6, which leads to an increase in the duration of the IL-6 signal during trans-signaling [11]. In our study, sIL-6Ra did not change plasma levels, while gp130 / sIL-6Rb decreased in patients with MetS 6.49 (4.44 - 37.62) ng / ml compared to the control group $13.9(10.9-29.4) \mathrm{ng} / \mathrm{ml}(\mathrm{p}<0.05)$. The level of soluble sIL-6Ra and gp130 / sIL-6Rb receptors was negatively correlated with BMI $(\mathrm{r}=-0.43, \mathrm{p}<0.05)(\mathrm{r}=-0.45, \mathrm{p}<0.05)$, with LDL level $(r=-0.33, p<0.05)(r=-0.30, p<0.05)$ and positively correlated with the level of $\operatorname{HDL}(\mathrm{r}=0.33, \mathrm{p}<0.05)$ $(\mathrm{r}=0.29, \mathrm{p}<0.05)$ in MetS patients (Figure 2). The level of gp130 / sIL-6Rb, but not the level of sIL-6Ra, negatively correlated with the level of glucose $(r=-0.29, p<0.05)$ in MetS patients (Figure 2).

\begin{tabular}{|c|c|c|c|c|c|c|c|c|}
\hline IL-6 & 0.11 & -0.02 & 0.56 & 0.50 & 0.29 & -0.19 & 0.15 & 0.11 \\
\hline IL-6Ra & -0.17 & -0.17 & -0.43 & -0.23 & 0.06 & 0.33 & -0.33 & 0.12 \\
\hline L-6Rb & -0.29 & -0.16 & -0.45 & -0.39 & -0.05 & 0.29 & -0.30 & 0.05 \\
\hline IL-11 & -0.18 & 0.13 & -0.14 & -0.16 & -0.04 & 0.17 & -0.23 & 0.04 \\
\hline CRP & 0.03 & 0.15 & -0.20 & -0.03 & -0.36 & -0.18 & -0.32 & -0.09 \\
\hline
\end{tabular}

Fig. 2. Correlation matrix of biochemical parameters and proinflammatory IL-6 family cytokines in patients with MetS

IL-6 level negatively correlated with sIL-6Ra $(r=-0.59)$ and gp130 / sIL-6Rb $(\mathrm{r}=-0.45)$ levels in MetS patients $(\mathrm{p}<$ $0.05)$. The increase in the level of IL- 6 in plasma was accompanied by a decrease in concentration gp 130 / sIL-6Rb, which is probably associated with impaired signaling of IL-6 and the development of metabolic complications.

IL-11 promotes fibrosis, steatosis, hepatocyte death, inflammation and hyperglycemia in mice with diet-induced steatohepatitis. Inhibition of IL-11 improved the cardiometabolic profile in animal models [12]. In contrast, other authors have shown that IL-11 inhibits NF-kappaB and AP-1 [13]. In this study, we recorded increased IL-11 loss in patients with MetS $0.57(0.44-1.01) \mathrm{pg} / \mathrm{ml}$ compared to a control of $0.18(0.12-0.48) \mathrm{pg} / \mathrm{ml}(\mathrm{p}<0.05)$. However, we did not find any correlation of IL-11 level with other studied parameters, which does not allow us to demonstrate its role in the pathogenesis of MetS.

\section{Conclusion}

Thus, a decrease in the concentration of soluble sIL-6Ra and gp130 / sIL-6Rb receptors was accompanied by increased dysregulation of carbohydrate and lipid metabolism.sIL-6Ra did not change, but was negatively correlated with BMI, LDL levels and HDL levels in blood plasma of patients with MetS. A decrease in the concentration of the soluble gp130/ sIL-6Rb receptor was accompanied by metabolic dysregulation: an increase in BMI, glucose levels, LDL levels and decrease in HDL levels in blood plasma of patients with MetS.

This fact may indicate the protective role of sIL-6Ra transsignaling and protective role of IL-6 signal inhibition due to high levels of sgp130 / sIL-6Rb in relation to metabolic disorders in obesity patients.

\section{ACKNOWLEDGMENT}

The research was supported by Russian Foundation for Basic Research [No. 18-015-00084-a to L.L. (patient recruitment and classification), by Russian Foundation for Basic Research and KLG [No.19-415-393004 - r_mol_a to D.S. (biochemical parameters), No.19-44-390005 - $\bar{r} \_$a to D.S. (flow fluorimetry)].

\section{REFERENCES}

[1] S. Swarup and R. Zeltser, Metabolic syndrome, StatPearls Publishing, 2020.

[2] C.A. Aguilar-Salinas and T. Viveros-Ruiz "Recent advances in managing/understanding the metabolic syndrome." F1000Res, vol. 8, April 2019

[3] D. Qu, J. Liu, C.W. Lau and Y. Huang "IL-6 in diabetes and cardiovascular complications.” Br. J. Pharmacol, August 2014, vol. 171(15), pp. 3595-3603.

[4] H.M. Sarbijani, M. Khoshnia and A. Marjani (2016) "The association between Metabolic Syndrome and serum levels of lipid peroxidation and interleukin-6 in Gorgan." Diabetes. Metab. Syndr, March 2016, vol. 10(1 Suppl 1), pp. 86-89.

[5] J.P. Bastard et al. "Elevated levels of interleukin 6 are reduced in serum and subcutaneous adipose tissue of obese women after weight loss." J. Clin. Endocrino.1 Metab, September 2000, vol. 85(9), pp. 3338-3342.

[6] S. Rose-John "Interleukin-6 Family Cytokines." Cold. Spring. Harb. Perspect Biol., February 2018, vol. 10(2).

[7] A.J. Covarrubias and T. Horng "IL-6 strikes a balance in metabolic inflammation.” Cell Metab., June 2014, vol. 19(6), pp. 898-899.

[8] S. Devaraj et al. (2008) "CRP and adiponectin and its oligomers in the metabolic syndrome: evaluation of new laboratory-based biomarkers." Am. J. Clin. Pathol., May 2008, vol. 129(5), pp. 815-822.

[9] M. Blüher et al. "Association of interleukin-6, C-reactive protein, interleukin-10 and adiponectin plasma concentrations with measures of obesity, insulin sensitivity and glucose metabolism." Exp. Clin. Endocrinol. Diabetes, October 2005, vol. 113(9), pp. 534-537.

[10] S. Shelbaya et al. "Study of the role of interleukin-6 and highly sensitive C-reactive protein in diabetic nephropathy in type 1 diabetic patients.” Eur. Rev. Med. Pharmacol. Sci., February 2012, vol. 16(2), pp.176-182.

[11] D. Schmidt-Arras and S. Rose-John "IL-6 pathway in the liver: From physiopathology to therapy." J. Hepatol., June 2016, vol. 64(6), pp. 1403-1415.

[12] A.A. Widjaja et al. (2019) "Inhibiting Interleukin 11 Signaling Reduces Hepatocyte Death and Liver Fibrosis, Inflammation, and Steatosis in Mouse Models of Nonalcoholic Steatohepatitis." Gastroenterology, September 2019, vol. 157(3), pp. 777-792.

[13] A. Lgssiar et al. "Interleukin-11 inhibits NF-kappaB and AP-1 activation in islets and prevents diabetes induced with streptozotocin in mice.” Exp. Biol. Med. (Maywood)., May 2004, vol. 229(5), pp. 425436 